\title{
Challenges posed by the scale-up of nanomedicines
}

\author{
"To face the challenges posed by the scale-up of nanomedicines, practical \\ experimental designs could provide efficient solutions. Once scale-up is achieved, \\ nanomedicines will be made better available in the market for the treatment of a \\ myriad of diseases."
}

\section{KEYWORDS: emulsion-based method - large-scale production - nanoprecipitation method - polymeric nanoparticles = solid lipid nanoparticles}

The recent cutting-edge developments in nanomedicines have brought numerous advances in the diagnosis and therapy of highly challenging diseases [1]. Nanomedicines, which are medical applications of nanotechnology, are mainly nanoparticle-based drug products. Several comprehensive definitions are now available for nanoparticles in nanomedicine. For pharmaceutical purposes, nanoparticles are colloidal particles that range in size from 10 to $1000 \mathrm{~nm}(1 \mu \mathrm{m})$, they consist of macromolecular materials in which the active principle (drug and/or diagnostic material) is dissolved, entrapped, encapsulated and/or to which the active principle is adsorbed or attached $[2,3]$. The various types of nanomedicines made are, for example, polymeric nanoparticles, solid lipid nanoparticles, liposomes, dendrimers, polymeric micelles and carbon nanotubes [2]. The US FDA-approved nanomedicine products, such as Doxil ${ }^{\circledR}$ (Janssen Products, LP, PA, USA) and Abraxane $^{\circledR}$ (Celgene Corporation, NJ, USA), are typical examples of the outcome of aggressive research on nanomedicines, and available in the market for the treatment of ovarian cancer and metastatic breast cancer, respectively [4]. However, nanomedicines still impose many challenges to researchers to obtain the maximum benefits for patients during diagnosis and therapy [5].

The launch of new nanomedicine products on the market is preceded by different developmental stages. A forthcoming major challenge among the developmental stages of nanomedicine is industrial scale-up (large scale industrial production). It is well known that nanomedicine preparations in the laboratory scale are achievable and well documented by various researchers [6,7]. At the same time, the industrial factorial design (scale-up) of nanomedicines has been considered less during development. The limited experiments and insufficient information on the scale-up technologies of nanomedicines may hinder the introduction of these nanopharmaceuticals into the market [8].

Although various methods are available for the production of nanomedicines, the two most familiar/major categories that require scale-up technologies are the emulsion-based method and nanoprecipitation method. The technical steps (e.g., introducing organic solvent into aqueous system, solvent evaporation, stirring rate and size reduction by forces) behind these two methods are similar to the steps of most of the other methods available for the fabrication of nanomedicines (e.g., solvent evaporation techniques, gelation technique and emulsion polymerization technique) $[8,9]$. Hence, this editorial focuses mainly on the challenges posed by the scale-up of the emulsion-based method and nanoprecipitation method used for the large-scale production of nanomedicines.

\section{Scale-up of nanomedicines: preparative methods \& their challenges}

The scale-up of nanomedicines includes the integration of methods as well as the transfer of technology for their large-scale industrial production. The process limitations in the smallscale preparation may lead to failure of translating any preparative method from a laboratory scale to an industrial scale. Also, a well-designed scale-up procedure will assure the quality of the nanomedicine, cost-effectiveness and a timely product launch. In the literature, there are only

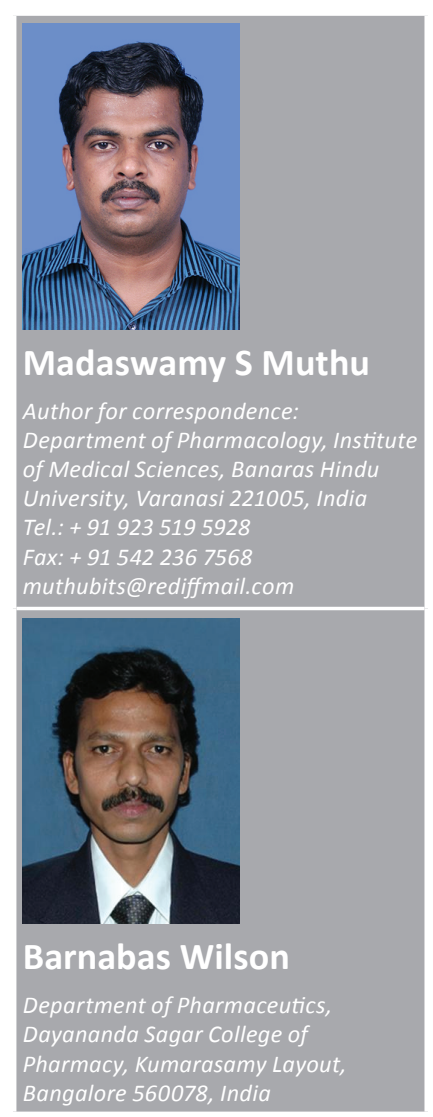

Future $\mathrm{fSO}$ 
a few reported laboratory-scale manufacturing processes of nanomedicines, especially using the emulsion-based and nanoprecipitation methods, which are supported by scale-up aspects for industrial manufacturing $[9,10]$.

The effects of scale-up and process limitations of preparative methods are considered to be major challenges during large-scale production.

First, scale-up has mainly been observed to affect the characteristics of nanoparticles, such as particle size, drug encapsulation, process residual materials, colloidal stability and surface morphology. Colombo et al. reported that the scale-up of the emulsion-based method, from the laboratory scale of $60 \mathrm{ml}$ to the industrial scale of 21 (nearly 33-fold), did not alter the encapsulation efficiency [11]. However, an increase in impeller speed and agitation time decreased the particle sizes. A decrease in the size of the nanoparticles was observed with a decrease in the polymer concentration. Another study by Galindo-Rodriguez et al. also reported that during scale-up, from a laboratory batch volume of $60 \mathrm{ml}$ to $1.5 \mathrm{l}$, increasing the stirring rate decreased the particle size in the emulsion-based method [9]. Moreover, they observed that the scale-up process reduced the drug loading of nanoparticles.

\section{"...a well-designed scale-up procedure will assure the quality of the nanomedicine, cost-effectiveness and a timely product launch."}

Indeed, Charcosset and Fessi, have investigated the possibility of scale-up for the nanoprecipitation method by a membrane contactor [10]. In this method, organic phase (containing hydrophobic materials) was introduced through the membrane pores via the filtrate side, to form droplets that react with the aqueous phase (containing surfactant), which can produce the nanoparticles. Also, it was observed in the membrane contactor method that the size of nanoparticles and the organic phase flux slightly decreased with an increase in crossflow velocity of the aqueous phase. However, for the nanoprecipitation method it showed high flux, which supports this method for the industrial scaleup of nanomedicines [10]. In one of the scaleup studies, the use of the membrane contactor method in the large-scale production of solid lipid nanoparticles was reported. The organic phase-containing lipid was pressed through the membrane pores, at a temperature above the melting point of the lipid. The lipid droplets formed were introduced into the aqueous phase flowing tangentially to the membrane. Finally, solid lipid nanoparticles were produced in large quantities when the nanosized lipid droplets were cooled below the lipid melting point [12]

Second, process limitations include the stability of materials used in the methods and toxic solvent usage (e.g., usage of chloroform or dichloromethane as organic phase). Mostly, owing to the toxic potential of organic solvents, pharmaceutical industries are now avoiding their usage even for the coating of tablets and granules by applying the aqueous coating method. Hence, novel methods using aqueous solvents or solvents with low toxicity have to be developed for the production of nanomedicines by the pharmaceutical industries [101].

Third, a short consumption time is always desired for a single pilot batch. GalindoRodriguez et al. compared the process time required for producing a pilot batch [9], and it was reported that the nanoprecipitation method had taken less time (120 min) when compared with the emulsion-based method (350 min) [9-12].

\section{Concluding remarks}

The approval of Doxil (doxorubicin liposomes) in 1995 and Abraxane (paclitaxel-bound albumin nanoparticles) in 2005 by the FDA and their subsequent commercialization for the treatment of ovarian cancer and breast cancer, respectively, confirms their importance/effectiveness in treating highly challenging as well as deadly diseases. In addition, a few advanced nanomedicine products (e.g., receptor-targeted nanomedicine) are in different phases of clinical trials [2]. Despite these potential achievements, nanomedicines suffer from challenges such as unexpected toxicities, their effect on the immune system, pharmaceutical stability issues and scale-up difficulties $[8,13]$. Among these, the clinical translation as well as medical application is mainly hindered by scale-up of nanomedicines. It appears from the literature search that not much attention has been paid for the issues related to the scale-up process. The success of any product, including nanomedicines, relies on its large-scale industrial production and commercialization. Therefore, the scientific community should pay more attention to the large-scale industrial production after their successful development of the laboratory scale. To face the challenges posed by the scale-up of nanomedicines, practical experimental designs could provide efficient solutions. Once scale-up 
is achieved, nanomedicines will be made better available in the market for the treatment of a myriad of diseases [14].

\section{Financial \& competing interests disclosure}

MS Muthu acknowledges the Department of Science and Technology, New Delhi, India, for the BOYSCAST
Fellowship (SR/BY/L-41/09). The authors have no other relevant affiliations or financial involvement with any organization or entity with a financial interest in or financial conflict with the subject matter or materials discussed in the manuscript apart from those disclosed.

No writing assistance was utilized in the production of this manuscript.

\section{References}

Papers of special note have been highlighted as: - of interest

1 Muthu MS, Rajesh CV, Mishra A, Singh S. Stimulus responsive targeted nanomicelles for effective cancer therapy. Nanomedicine (Lond.) 4(6), 657-667 (2009).

2 Muthu MS, Singh S. Targeted nanomedicines: effective treatment modalities for cancer, AIDS and brain disorders. Nanomedicine (Lond.) 4(1), 105-118 (2009).

- Describes the types of nanomedicines and their role for future therapy.

3 Muthu MS, Singh S. Studies on biodegradable polymeric nanoparticles of risperidone: in vitro and in vivo evaluation. Nanomedicine (Lond.) 3(3), 305-319 (2008).

4 Muthu MS, Feng SS. Nanopharmacology of liposomes developed for cancer therapy. Nanomedicine (Lond.) 5(7), 1017-1019 (2010).

5 Muthu MS, Wilson B. Multifunctional radionanomedicine: a novel platform for effective cancer imaging and therapy. Nanomedicine (Lond.) 5(2), 169-171 (2010).

6 Kulkarni SA, Feng SS. Effect of surface modification on delivery efficiency of biodegradable nanoparticles across the blood-brain barrier. Nanomedicine (Lond.) 6(2), 377-394 (2011)

7 Muthu MS, Kulkarni SA, Raju A, Feng SS. Theranostic liposomes of TPGS coating for targeted co-delivery of docetaxel and quantum dots. Biomaterials doi:10.1016/j. biomaterials.2012.01.036 (2012) (In Press).

8 Saraf S. Process optimization for the production of nanoparticles for drug delivery applications. Expert Opin. Drug Deliv. 6, 187-196 (2009).

9 Galindo-Rodriquez S, Puel F, Briancon S, Allemann E, Doelker E, Fessi H. Comparative scale-up of three methods for producing ibuprofen-loaded nanoparticles. Eur. J. Pharm. Sci. 25, 357-367 (2005).

10 Charcosset C, Fessi H. A new process for drug loaded nanocapsules preparation using a membrane contactor. Drug Dev. Ind. Pharm. 31, 987-992 (2005).
11 Colombo AP, Briancon S, Lieto J, Fessi H. Project, design, and use of a pilot plant for nanocapsule production. Drug Dev. Ind. Pharm. 27, 1063-1072 (2001).

12 El-Harati AA, Charcosset C, Fessi H. Influence of the formulation for solid lipid nanoparticles prepared with a membrane contactor. Pharm. Dev. Technol. 11, 153-157 (2006).

13 Muthu MS, Feng SS. Pharmaceutical stability aspects of nanomedicines. Nanomedicine (Lond.) 4(8), 857-860 (2009).

14 Wilson B. Therapeutic compliance of nanomedicine in Alzheimer's disease. Nanomedicine (Lond.) 6(7), 1137-1139 (2011).

\section{Website}

101 International Conference on Harmonisation Q3C (R5). Guideline for Residual Solvents. ICH Step 4 version dated February 2011. www.ich.org/fileadmin/Public_Web_Site/ ICH_Products/Guidelines/Quality/Q3C/ Step4/Q3C_R5_Step4.pdf 